\title{
A Comparison of Osseous and Extraosseous Ewing Sarcoma
}

\author{
Selin Akturk Esen ${ }^{1}$, Yusuf Acikgoz ${ }^{1}$, Oznur Bal ${ }^{1}$, Birol Yildiz², Gokhan Ucar ${ }^{1}$, Merve Dirikoc ${ }^{1}$, Yakup Ergun ${ }^{1}$, Efnan \\ Algin $^{1}$ and Dogan Uncu ${ }^{1}$ \\ ${ }^{1}$ Department of Medical Oncology, Health Sciences University, Ankara City Hospital, Ankara, Turkey \\ ${ }^{2}$ Department of Medical Oncology, Elazığ Fethi Sekin City Hospital, Turkey
}

\begin{abstract}
Objective: To compare the clinicopathological characteristics, treatment responses, survival analysis of osseous Ewing sarcoma (OES) and extraosseous ES (EES).

Study Design: Observational study.

Place and Duration of Study: Ankara City Hospital and Ankara Numune Training Research Hospital Medical Oncology Clinics from January 2005 to February 2020.

Methodology: Clinicopathological characteristics of histologically confirmed ES/PNET and followed up, and treatment modalities were recorded from patients' registration data-base of the hospital. Lactate dehydrogenase (LDH), alkaline phosphatase (ALP), hemoglobin were measured before chemotherapy or surgery. The patients with a second cancer, gall bladder/biliary tract diseases, viral hepatitis and other bone diseases were excluded.

Results: Sixty seven patients evaluated retrospectively. Out of the total patients, $56.7 \%$ consisted of OES, and $43.3 \%$ consisted of EES. The median age of the EES group (26 years) was significantly higher than that of the OES group ( 22 years, $p=0.008$ ). The most common metastasis region was lung in both the groups. Age, LDH levels and stage of the disease were found to be statistically significant prognostic factors in univariate and multivariate analysis. The median OS of patients who started with local treatment (surgical, surgical \pm radiotherapy) and followed up with chemotherapy was 82.6 months (95\% Cl, 55.2-110.1), while the median OS of patients who received local treatment between or after chemotherapy was 43.4 months ( $95 \% \mathrm{Cl}$, 13.2-73.6, $p=0.042$ ).

Conclusion: Patients with extrosseus ES were significantly older. Age, LDH levels, stage of disease, local treatment followed by systemic therapy are important associated factors.
\end{abstract}

Key Words: Osseous ewing sarcoma, Extraosseous ewing sarcoma, Chemotherapy, Local treatment.

How to cite this article: Esen SA, Acikgoz Y, Bal O, Yildiz B, Ucar G, Dirikoc M, Ergun Y, Algin E, Uncu D. A Comparison of Osseous and Extraosseous Ewing Sarcoma. J Coll Physicians Surg Pak 2021; 31(01):27-33.

\section{INTRODUCTION}

Ewing sarcoma (ES) and primitive neuroectodermal tumor (PNET) are parts of a spectrum of neoplastic diseases known as the ES family of tumors (EFT). ${ }^{1}$ EFT also includes extraosseous ES (EES), atypical ES, malignant small cell tumors of thoracopulmonary region (Askin tumor). ${ }^{2}$ They have similar histologic, immunohistochemical characteristics and nonrandom chromosomal translocations; ${ }^{3}$ so these tumors are considered to be derived from a common cell of origin. ${ }^{4}$

Correspondence to: Dr. Selİn Akturk Esen, Department of Medical Oncology, Health Sciences University Ankara City Hospital, Turkey

E-mail:drselin16@hotmail.com

Received: August 31, 2020; Revised: December 15, 2020;

Accepted: December 22, 2020

DOI: https://doi.org/10.29271/jcpsp.2021.01.27
While most of the ES cases present with localised disease at the time of diagnosis, $20-25 \%$ of them present with metastasis. ${ }^{5}$ Most of the cases develop in the skeletal system in childhood, $20-30 \%$ of them originate from the extraskeletal regions. ${ }^{6}$ The incidence of EES is 1 per 5-10 million. ${ }^{7}$ The median age at diagnosis for skeletal cases is younger than that of extraskeleteal cases. $^{8}$

Standard treatment of ES consists of chemotherapy and local therapies including surgery and/or radiotherapy. ${ }^{9}$ Complete surgical resection is a preferred local control method but radiotherapy is an alternative for tumors that cannot be resected or patients who refuse surgery. ${ }^{9}$ While EES was previously treated as rhabdomyosarcoma, studies have shown that these patients respond to OES treatment protocols. ${ }^{10}$

There are several studies comparing OES and EES tumor characteristics and disease outcomes. Whether disease prognostic factors, treatment responses, and survival rates are similar in OES and EES remains controversial. In addition, there is not 
enough evidence showing that EES chemotherapy protocols are the most appropriate treatmentforOES.

This study was carried out to compare the disease clinicopathological characteristics, treatment responses, survival analysis of OES and EES cases; and to contribute to the literature on these controversial issues.

\section{METHODOLOGY}

This study was approved by Institutional Ethics Committee of Ankara City Hospital (E1/537/2020). The patients with histologically confirmed ES/PNET and followed up in Hospital between January 2005 and February 2020 were included in this retrospective study. Clinicopathological characteristics of the patients and treatment modalities were recorded from patients' registration data-base of the hospital. Lactate dehydrogenase (LDH), alkaline phosphatase (ALP), hemoglobin $(\mathrm{Hb})$, and erythrocyte sedimentation rate (ESR) were measured before treatment (chemotherapy or surgery). The patients with a second cancer, gall bladder/biliary tract diseases, viral hepatitis, and other bone diseases were excluded from the study.

Statistical analyses were performed by using SPSS Statistics version 24.0 (IBM Corp., Chicago). Continuous variables (age, tumor size, duration of treatment, LDH, ALP, Hb, ESR levels) were expressed as either mean \pm S.D or median with 25th percentile and 75th percentile. Categorical variables (gender, ECOG PS, tumor lacation, metastatic site, chemotherapy regimen, and pathological staining) were presented as percentage. Normality of quantitative data has been analysed by Kolmogorov-Smirnov and Shapiro-Wilk tests. Optimal cut-off value of numerical prognostic variable (LDH) was determined calculating area under the curve (AUC) of Receiver Operating Characteristic (ROC) analysis. The maximal joint point of sensitivity and specificity was calculated by the Youden Index. Pearson Chi-squaretest was used for the comparison of categorical variables of two groups, and independent sample T-test or Mann-Whitney U-test was used for comparison of continuous variables of two groups. Survival analysis was calculated according to Kaplan-Meier (Log rank, Breslow and Tarone-Ware analyses) method. Univariate Cox regression analysis was performed in order to determine the independent predictors on overall survival (OS) and progression-free survival (PFS). Variables, which have a p-value less than 0.05 in univariate analysis, were put into multivariate analysis. P-value $<0.05$ was considered asstatistically significant.

\section{RESULTS}

Sixty-seven patients evaluated retrospectively in this study. Out of the total patients, $56.7 \%(n=38)$ patients consisted of OES, and $43.3 \%(n=29)$ consisted of EES. Moreover, $83.6 \%$ of all ES were typical ES. While $81.6 \%$ of the OES group was typical ES and $10.5 \%$ was atypical ES, $86.2 \%$ of the EES group was typical ES and 10.3 was atypical ES $(p=0.746)$. The median age of the EES group (26 years) was statistically significantly higher than that of the OES group ( 22 years, $p=0.008$ ). Tumor size was
$7.7 \pm 3.5 \mathrm{~cm}$ in the OES group and $9.5 \pm 5.5 \mathrm{~cm}$ in the EES group ( $p=0.119$ ). In $46.3 \%$ of the whole group (OES + EES), the tumor was located in the trunk and $32.8 \%$ in the lower extremities. Tumor localisation for OES was $50 \%$ in the lower extremities and $23.7 \%$ in the trunk. On the other hand, $75.9 \%$ EES was located in the trunk, while it waslocated on the lower extremities and head-neck ( $10.3 \%$ and $10.3 \%$, respectively) in the second frequency $(p<0.001)$.

Pathologically, $38.8 \%$ of the entire ES group was stained with CD99, $19.4 \%$ with vimentin and $11.9 \%$ with FLI-1. There was no significant difference between EES and OES groups in terms of CD99, vimentin and FLI-1 staining ( $p=0.446, p=0.418, p=$ 0.076 , respectively).

While $71.1 \%$ ( $n=27$ ) of OES patients received chemotherapy as the first treatment, $58.6 \%(n=17)$ of EES patients were surgically treated ( $p=0.015$ ). Chemotherapy regimens given as initial therapy or after surgery, were similar.

There was no difference between the two groups in terms of gender, eastern cooperative oncology group (ECOG) performance status, tumor size, pathological subtype, disease stage, metastatic region, treatment response, LDH levels, ALP levels, hemoglobin levels, neutrophil lymphocyte ratio (NLR), platelet lymphocyte ratio (PLR), and erythrocyte sedimentation rate (ESR, Tablel).

Age, LDH levels and stage of the disease werefound to bestatistically significant prognostic factors in univariate analysis. These factors were also statistically significant prognostic factors for OS in multivariate analysis (Table II).

LDH was found to be a prognostically significant laboratory parameter for ES. ROC analysis was applied to determine the LDH cut-off value. The ROC analysis was performed by using survival status as an end-point and calculated AUC was 0.67 for LDH (95\% Cl: 0.54-0.81, p = 0.016). The recommended cut-off value was 320.5 for LDH with $64.9 \%$ senistivity and $72.4 \%$ sepcifity. Patients were stratified into two groups according to cut-off values of LDH as follows: $\mathrm{LDH}<320.5 \mathrm{U} / \mathrm{L}$ (normal) and $\mathrm{LDH} \geq 320.5 \mathrm{U} / \mathrm{L}$ (high).

There was no statistically significant difference between the OES and EES groups in terms of OS. The median OS was 82.6 (59.6-105.7) months for OES patients, and 36.6 (9.1-64.1) months for EES patients ( $p=0.258$ ). There was no statistically significant difference between the OES and EES groups in terms of PFS. The median PFS was 46.6 (NA) months in the OES group and 20.7 (8.1-33.3) months in the EES group ( $p=0.287$ ). At the beginning of the treatment or after chemotherapy, 41 of our patients received radiotherapy and 21 received surgical \pm radiotherapy as local treatment. The median OS of patients whostarted with local treatment (surgical, surgical \pm radiotherapy) and followed up with chemotherapy was 82.6 months (95\% Cl, 55.2-110.1), while the median OS of patients who received local treatment between or after chemotherapy was 43.4 months $(95 \% \mathrm{Cl}, 13.2-73.6, \mathrm{p}=0.042)$. 
Table I: Baseline clinicopathological characteristics of patients.

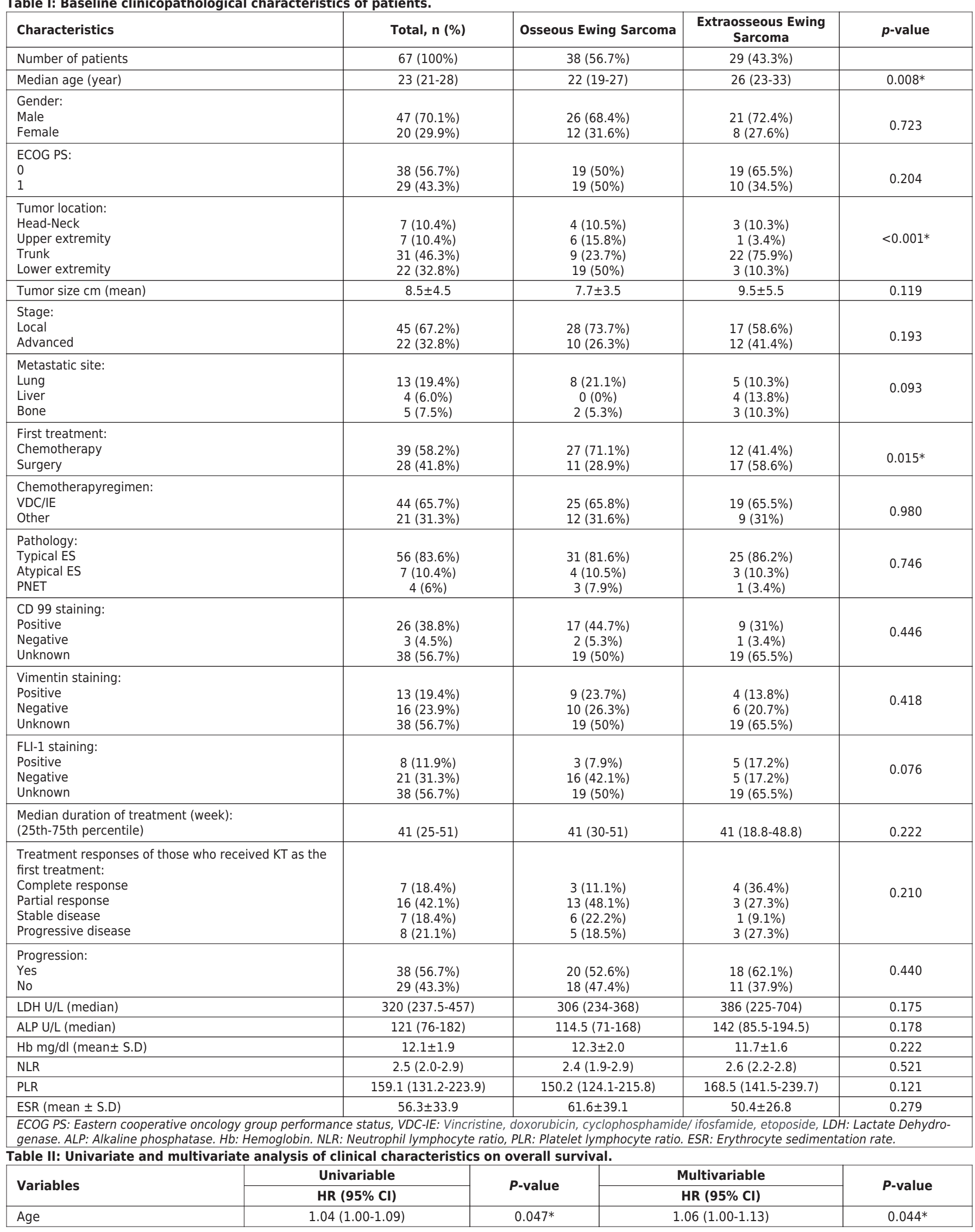




\begin{tabular}{|c|c|c|c|c|}
\hline $\begin{array}{l}\text { Gender: } \\
\text { Male } \\
\text { Female }\end{array}$ & $\begin{array}{c}1 \\
1.33(0.67-2.68)\end{array}$ & 0.414 & $\begin{array}{c}1 \\
1.58(0.65-3.83)\end{array}$ & 0.313 \\
\hline $\begin{array}{l}\text { ECOG PS: } \\
0 \\
1\end{array}$ & $\begin{array}{c}1 \\
1.27(0.66-2.43)\end{array}$ & 0.471 & $\begin{array}{c}1 \\
1.62(0.72-3.64)\end{array}$ & 0.241 \\
\hline $\begin{array}{l}\text { Tumor location: } \\
\text { Head-Neck } \\
\text { Extremity } \\
\text { Trunk }\end{array}$ & $\begin{array}{c}1 \\
0.72(0.27-1.97) \\
0.66(0.24-1.83)\end{array}$ & 0.729 & $\begin{array}{c}1 \\
1.09(0.31-3.85) \\
0.41(0.14-1.22)\end{array}$ & 0.081 \\
\hline $\begin{array}{l}\text { Pathology: } \\
\text { Typical ES } \\
\text { Atypical ES } \\
\text { PNET }\end{array}$ & $\begin{array}{c}1 \\
0.68(0.16-2.85) \\
1.03(0.25-4.33) \\
\end{array}$ & 0.869 & $\begin{array}{c}1 \\
0.56(0.11-2.75) \\
1.33(0.26-6.85) \\
\end{array}$ & 0.740 \\
\hline $\begin{array}{l}\text { Origination site: } \\
\text { Osseous } \\
\text { Extraosseous }\end{array}$ & $\begin{array}{c}1 \\
1.45(0.76-2.79)\end{array}$ & 0.260 & $\begin{array}{c}1 \\
1.63(0.66-4.06)\end{array}$ & 0.293 \\
\hline $\begin{array}{l}\text { LDH level: } \\
\text { Normal } \\
\text { High }\end{array}$ & $\begin{array}{c}1 \\
2.27(1.15-4.47\end{array}$ & $0.018 *$ & $\begin{array}{c}1 \\
2.41(1.11-5.22)\end{array}$ & $0.026 *$ \\
\hline $\begin{array}{l}\text { Stage: } \\
\text { Local } \\
\text { Advanced }\end{array}$ & $\begin{array}{c}1 \\
2.09(1.08-4.02)\end{array}$ & $0.028^{*}$ & $\frac{1}{2.15(1.06-4.35)}$ & $0.034 *$ \\
\hline $\begin{array}{l}\text { Chemotherapy regimen: } \\
\text { VDC/IE } \\
\text { Others }\end{array}$ & $\begin{array}{c}1 \\
1.34(0.69-2.59)\end{array}$ & 0.388 & $\begin{array}{c}1 \\
1.03(0.43-2.45)\end{array}$ & 0.954 \\
\hline
\end{tabular}

\section{DISCUSSION}

In this study, the differences in disease presentations between OES and EES were investigated. Median age of OES was statistically significantly lower than EES and male gender was more dominant in both the groups. PNET was the least common pathological subgroup in all ES groups. Local disease was more common in both OES and EES patients. All these findings were consistent with previous studies. $^{1,8}$

There are different results in different studies on ES primary site. In OES patients, tumor was mostly located on lower extremities (totally $67.2 \%$ were localised in the upper-lower extremities and $32.8 \%$ in the trunk and head- neck in OES patients); but in EES patients, it was mostly located on trunk. However, Jiang et al. observed that OES tumor was localised on axial skeleton at a rate of $52.8 \%$ and on appendicular skeleton at a rate of $43.9 \%$ in their study, which was different from this study. ${ }^{11}$ But similar to the present study, in the compilation of data from 975 patients from the European intergroup cooperative Ewing sarcoma studies (EICESS),

the distribution of primary areas is found in $54 \%$ axial skeleton and $42 \%$ appendicular skeleton. ${ }^{12}$ Similar to these results, Orr et al. showed that tumor of EES was located in trunk, extremities (lower and upper) and head-neck, respectively. ${ }^{13}$ There was no difference in tumor size between EES and OES patients. Different data are available in different studies on this subject. Similar to this study, some studies showed that there was no difference in tumor size between the two groups; others showed that EES tumors were smaller at diagnosis. ${ }^{6,11}$ In this study, while $81.6 \%$ of the OES group was typical ES and $10.5 \%$ was atypical ES, $86.2 \%$ of the EES group was typical ES and $10.3 \%$ was atypical ES.

Most of ES tumors express CD99, which is a highly sensitive immunohistochemical biomarker. CD57, synaptophysin, chromogranin, vimentin, neuron-specific enolase and S-100 are often expressed in ES. ${ }^{14}$ Antibody against FLI-1, which is centered in the nucleus of the tumor cells, has been shown to be specific for EFT. ${ }^{15}$ In this study, the most positively detected markers in pathological staining were CD99, vimentin and $\mathrm{FLI}-1$, respectively. There were no difference between OES and EES groups in terms of pathological staining.

The most common distant metastasis site was lung in both OES and EES patients. In OES patients, distant metastases were seen in bones with the second frequency. However, in EES patients, liver was the second most common distant metastasis site. Metastatic spreading pattern may be different between EES and OES. Worch et al. detected that tumor spreading sites were lung, bone and bone marrow, respectively in OES patients. ${ }^{16}$ Another study showed that lymph nodes were the most frequent metastatic site, followed by lungs, bones, solid organs, peritoneum and pleura in EES patients. ${ }^{17}$ Since the authors did not include lymph node metastasis as a distant metastasis site, it might be the reason for the most common metastasis site to be lung in this study.

Although there was a numerical difference for OS and PFS between OES and EES groups, it did not reach statistical significance. There may be two reasons for OS for this situation. First of all, in Kaplan-Meier analysis for OS, it is seen that the curves diverged from each other at 24 months but 
converged at 96 months. This situation can cause a numerical difference. The second reason may be the relatively small number of patients analysed statistically. In the analysis for PFS, the median value could not be reached in the OES group. Therefore, the numerical difference did not reach statistical significance. So, different results can be obtained in more mature analyses.

The median OS was statistically better in the group which received local treatment (surgical \pm radiotherapy) and followed up with chemotherapy than the group received local treatment between or after chemotherapy. All of the patients who started with local treatment were first operated. Moreover, $58.6 \%$ of these patients were EES. The authors could not find a study comparing local treatment before and after chemotherapy. It is well known that chemotherapy is the mainstay of treatment in ES and is a necessary addition to local control to achieve a reasonable expectation of cure. ${ }^{18}$ ES treatment can be reevaluated in this respect with studies with large patient populations that will be divided into two groups as OES and EES patients. Since five patients could only receive chemotherapy due to their widespread metastases; and there was no patient who had only surgical treatment, one could not compare the patients received chemotherapy + local treatment with those received only chemotherapy or only surgical treatment. Previous studie shows that results are best when chemotherapy is combined with optimal local therapy, including radiation and/or surgical resection. ${ }^{19}$ The reduction of local tumor volume is accomplished in the majority of the patients; this may facilitate resection and reduce mortality and morbidity. Tumor localisation should be considered when making surgery decision in ES patients. Especially, the risk of surgical morbidity in pelvic OES tumors is higher than other localisations. ${ }^{20}$ In addition, resection of bone tumors in OES patients has higher morbidity than resection of EES soft tissue tumors. ${ }^{21}$ In OES, chemotherapy may be considered first to reduce morbidity as in pelvic tumors; and to shrink the tumor before surgery, as in extremity bone tumors.

In the present study, age, LDH levels and tumor stage were statistically significant prognostic factors for OS among all patients on univariate analysis and multivariate analysis. Tumor location, origin or chemotherapy regimen were not prognostic factors for OS. In another study, age, tumor size, tumor stage, and surgery were found to be factors that significantly associated with OS. $^{11}$ A different study showed that age, sex, tumor size and stage correlated with survival. ${ }^{8}$

In this study, optimal cut-off value for LDH level was found as 320.5 in ROC analysis for LDH. LDH $<320.5 \mathrm{U} / \mathrm{L}$ was considered normal and LDH> $320.5 \mathrm{U} / \mathrm{L}$ was considered high in ES patients. LDH level was also found to be a significant prognostic factor for OS in ES patients. LDH isoforms that contain predominantly $M$ subunits ( $\mathrm{LDH}-\mathrm{A}$ ) rises in many types of cancer and is linked to tumor growth, maintenance and invasion. ${ }^{22}$ In a study investigating the role of LDH-A in hepatocel- lular cancer (HCC) metastasis, HCC cell lines have been shown to over-express LDH-A and LDH-A inhibition increases apoptosis by the production of reactive oxygen species. ${ }^{23}$ Besides, the breakdown of LDH-A caused a significant decrease in metastatic potential. ${ }^{23}$ In a study examining the relationship between breast cancer and LDH, LDH-A expression was found to be an independent factor that strongly correlated with tumor size. ${ }^{24}$ In the same study, down regulation of LDH-A led to Ki67 reduction and induction of tumor cell apoptosis. In a study investigating the relationship between ES and LDH, it has been shown that genetic or pharmacological inhibition of LDH-A reduces tumor cell proliferation, induces apoptosis, and is associated with suppression of glycolytic flux and impairment of NADH / NAD + ratio. ${ }^{25}$

There were several limitations of this study. First, the number of patients was relatively small. Second, since it is a retrospective study using the hospital database, data related to external factors that may affect the prognosis of the disease and the toxicities developing in patients during the treatment process could not be obtained. Third, more importantly, the lack of regional lymph node metastasis information was an important limitation.

\section{CONCLUSION}

The prognostic factors, clinicopathological and treatment differences between OES and EES patients were evaluated in this study. Median age was higher in the EES group. Male gender was more dominant in both groups. Lung was most frequent distant organ metastasis site in both OES and EES groups. There was no OS and PFS differences between the two groups. Median OS was better in the group that started with local therapy than the group that started with chemotherapy. There is a requirement for studies involving larger patient populations, different ethnic groups, and investigating the adults' outcomes of ES, which is known to be seen more frequently in the pediatric population.

\section{ETHICAL APPROVAL:}

This study was approved by Institutional Ethics Committee of Ankara City Hospital (E1/537/2020).

\section{PATIENTS' CONSENT:}

Because this study was retrospective, the patients' consents were waived.

\section{CONFLICT OF INTEREST:}

All authors declared no conflict of interest.

\section{AUTHORS' CONTRIBUTION:}

SAE, YA, OB, GU, YE, BY, MD, DU, EA: Conception of the work, analysis or interpretation of data for the work, and drafting the work.

\section{REFERENCES}

1. Lee JA, Kim DH, Lim JS, Koh JS, Kim MS, Kong CB, et al. Soft- 
-tissue Ewing sarcoma in a low-incidence population: comparison to skeletal Ewing sarcoma for clinical characteristics and treatment outcome. Jpn J Clin Oncol 2010; 40(11):1060-7. DOI: 10.1093/jjco/hyq080.

2. Granowetter $L$ and West DC. The Ewing's sarcoma family of tumors: Ewing's sarcoma and peripheral primitive neuroectodermal tumor of bone and soft tissue. Cancer Treat Res 1997; 92: 253-308. DOI: 10.1007/978-1-4615-5767-8_9.

3. Ambros IM, Ambros PF, Strehl S, Kovar H, Gadner H, Kuntschik MS. MIC2 is a specific marker for Ewing's sarcoma and peripheral primitive neuroectodermal tumors. Evidence for a common histogenesis of Ewing's sarcoma and peripheral primitive neuroectodermal tumors from MIC2 expression and specific chromosome aberration. Cancer 1991; 67(7):1886-93. DOI: 10.1002/1097-0142 (19910401)67:7<1886::aid-cncr2820670712>3.0.c0;2-u.

4. Tirode F, Laud-Duval K, Prieur A, Delorme B, Charbord P, Delattre 0 . Mesenchymal stem cell features of Ewing tumors. Cancer Cell 2007; 11(5): 421-9. DOI: 10.1016/j.Ccr.2007.02.027.

5. Khanna N, Pandey A, Bajpai J. Metastatic ewing's sarcoma: Revisiting the "Evidence on the fence". Indian J Med Paediatr Oncol 2017; 38(2):173-81. 2017/09/14. DOI: 10.4103/ijmpo.ijmpo_24_17.

6. Applebaum MA, Worch J, Matthay KK, Goldsby RR, Neuhaus J, West DC, et al. Clinical features and outcomes in patients with extraskeletal Ewing sarcoma. Cancer 2011; 117(13):3027-32. 2011/06/22. DOI: 10.1002/cncr.25840.

7. Siegel RL, Miller KD, Jemal A. Cancer statistics, 2018. CA Cancer J Clin 2018; 68:7-30. DOI: 10.3322/caac.21442.

8. Lynch AD, Gani F, Meyer CF, Morris CD, Ahuja N, Johnston FM. Extraskeletal versus Skeletal Ewing Sarcoma in the adult population: Controversies in care. Surg Oncol 2018; 27(3): 373-379. DOI: 10.1016/j.suronc.2018.05.016.

9. Becker RG, Gregianin LJ, Galia CR, Jesus-Garcia Filho R, Toller EA, Badell G, et al. What is the impact of local control in Ewing sarcoma: Analysis of the first Brazilian collaborative study group - EWING1. BMC Cancer 2017; 17(1):420. DOI: 10.1186/s12885-017-3391-5.

10. Leachman BK and Galloway TJ. The role for radiation therapy in the management of sarcoma. Surg Clin North Am 2016; 96(5):1127-39. DOI: 10.1016/j.suc.2016.05.003.

11. Jiang S, Wang G, Chen J, Dong Y. Comparison of clinical features and outcomes in patients with extraskeletal vs skeletal Ewing sarcoma: An SEER database analysis of 3,178 cases. Cancer Manag Res 2018; 10: 6227-6236. DOI: 10.2147/CMAR.S178979.

12. Cotterill SJ, Ahrens S, Paulussen M, Jürgens, HF, Voûte PA, Gadner $\mathrm{H}$, et al. Prognostic factors in Ewing's tumor of bone: Analysis of 975 patients from the European Intergroup Cooperative Ewing's Sarcoma Study Group. J Clin Oncol 2000; 18(17):3108-114. DOI: 10.1200/JCO.2000.18.17.3108.

13. Orr WS, Denbo JW, Billups CA, Wu J, Navid F, Rao BN, et al. Analysis of prognostic factors in extraosseous Ewing sarcoma family of tumors: review of St. Jude Children's Research Hospital experience. Ann Surg Oncol 2012; 19(12):3816-22. DOI: 10.1245/s10434-012-2458-4.
14. Fletcher $C D$. The evolving classification of soft tissue tumours - an update based on the new 2013 WHO classification. Histopathol 2014; 64(1):2-11. DOI: 10.1111/his.12267.

15. Nilsson G, Wang M, Wejde J, Kreicbergs A, Larsson O. Detection of EWS/FLI-1 by Immunostaining. An adjunctive tool in diagnosis of ewing's sarcoma and primitive neuroectodermal tumour on cytological samples and paraffinembedded archival material. Sarcoma 1999; 3(1):25-32. DOI: 10.1080/13577149977839.

16. Worch J, Ranft A, DuBois SG, Paulussen M, Juergens $H$, Dirksen $U$. Age dependency of primary tumor sites and metastases in patients with Ewing sarcoma. Pediatr Blood Cancer 2018; 65(9):e27251. DOI: 10.1002/pbc.27251.

17. Huh J, Kim KW, Park SJ, Kim HJ, Lee JS, Tirumani SH, et al. Imaging features of primary tumors and metastatic patterns of the extraskeletal ewing sarcoma family of tumors in adults: A 17-year experience at a single institution. Korean J Radiol 2015; 16(4):783-90. DOI: 10.3348/kjr.2015.16.4.783.

18. Nesbit ME Jr, Gehan EA, Burgert EO Jr, Vietti TJ, Cangir A, Tefft $M$, et al. Multimodal therapy for the management of primary, nonmetastatic Ewing's sarcoma of bone: A longterm follow-up of the First Intergroup study. J Clin Oncol 1990; 8(10):1664-74. DOI: 10.1200/JCO.1990.8.10.1664.

19. Raciborska A, Bilska K, Rychlowska-Pruszynska M, Duczkowski M, Duczkowska A, Drabko k, et al. Management and follow-up of Ewing sarcoma patients with isolated lung metastases. J Pediatr Surg 2016; 51(7):1067-71. DOI: 10.1016/j.jpedsurg.2015.11.012.

20. Gaspar N, Hawkins DS, Dirksen U, Lewis LJ, Ferrari S, Le Deley MC, et al. Ewing sarcoma: Current management and future approaches through collaboration. J Clin Oncol 2015; 33(27):3036-46. 2015/08/26. DOI: 10.1200/JCO.2014. 59.5256.

21. Stokke J, Sung L, Gupta A, Lindberg A, Rosenberg AR. Systematic review and meta-analysis of objective and subjective quality of life among pediatric, adolescent, and young adult bone tumor survivors. Pediatr Blood Cancer 2015; 62(9): 1616-29. DOI: 10.1002/pbc.25514.

22. Gallo M, Sapio L, Spina A, Naviglio D, Calogero A, Naviglio S. Lactic dehydrogenase and cancer: an overview. Front Biosci (Landmark Ed) 2015; 20:1234-1249. DOI: 10.2741/4368.

23. Sheng SL, Liu JJ, Dai YH, Sun XG, Xiong XP, Huang G. Knockdown of lactate dehydrogenase A suppresses tumor growth and metastasis of human hepatocellular carcinoma. FEBS J 2012; 279(20):3898-3910. DOI: 10.1111/j.1742-4658. 2012.08748.x.

24. Wang ZY, Loo TY, Shen JG, Wang N, Wang DM, Yang DP, et al. LDH-A silencing suppresses breast cancer tumorigenicity through induction of oxidative stress mediated mitochondrial pathway apoptosis. Breast Cancer Res Treat 2012; 131(3):791-800. DOI: 10.1007/s10549-011-1466-6.

25. Yeung C, Gibson AE, Issaq SH, Oshima N, Baumgart JT, Edessa LD, et al. Targeting glycolysis through inhibition of lactate dehydrogenase Impairs tumor growth in preclinical models of ewing sarcoma. Cancer Res 2019; 79(90): 5060-5073. 2019/08/23. DOI: 10.1158/0008-5472.CAN19-0217. 
$\bullet \bullet \bullet \bullet \bullet \bullet \bullet \bullet \bullet \bullet$ 